Verzichtbar sind die - inhaltlich nicht überzeugenden - Ausführungen zur rechtlichen Einordnung des Auseinanderbrechens der Sowjetunion und Jugoslawiens. Zwar behauptet die Autorin eingangs, daß für Dismembration und Sezession möglicherweise unterschiedliche Regeln bezüglich des Wechsels der Staatsangehörigkeit gelten; in ihren Ergebnissen schlägt sich das aber nicht nieder. In diesem Zusammenhang wird die Resolution der Generalversammlung zur Mitgliedschaft Jugoslawiens in den Vereinten Nationen mit ihren Abweichungen gegenüber der entsprechenden Resolution des Sicherheitsrats mißdeutet. Falsch ist es weiterhin, die u.a. vom Rezensenten vertretene Auffassung, für die Einordnung einer Staatensukzession komme es wesentlich auf die Einschätzung der Staatengemeinschaft an, mit der deklaratorischen Anerkennungstheorie zu vermengen. Die Anerkennung als Staat und die Bewertung des Entstehungsvorgangs als Dismembration oder Sezession sind zwei verschiedene Paar Schuhe.

Löblich ist die Aufnahme eines Sachregisters, ärgerlich, daß man von den angegebenen Seitenzahlen stets zwei bis drei abziehen muß, um fündig zu werden. Nicht auf das Konto der Autorin geht, daß die Konvention des Europarats über die Staatsangehörigkeit von 1997 nur im Entwurf behandelt wird - sie hat ihr Manuskript bereits vorher abgeschlossen.

Ulrich Fastenrath

\title{
Michael Fraas
}

\section{Sicherheitsrat der Vereinten Nationen und Internationaler Gerichtshof}

Die Rechtmäßigkeitsprüfung von Beschlüssen des Sicherheitsrats der Vereinten Nationen im Rahmen des VII. Kapitels der Charta durch den Internationalen Gerichtshof Peter Lang Verlag, Frankfurt/Main, 1998, 258 S., DM 98,--

Mit dem Ende des Kalten Krieges hat die Frage der Kontrolle des Sicherheitsrats erheblich an Gewicht gewonnen. War der Sicherheitsrat bis 1990 weitgehend durch das Veto der sich gegenüberstehenden Blöcke in seiner Tätigkeit gehindert, änderte sich dies entscheidend mit dem Ende des Ost-West-Konflikts. Mit dem Ende dieses Konflikts fand der Sicherheitsrat sich in der Lage, in bisher nicht gekanntem Maße auf Krisen zu reagieren. Diese Neubelebung des Rates hat unter anderem die Frage nach einer gerichtlichen Kontrolle aufgeworfen. Die Arbeit von Michael Fraas befaßt sich mit dieser Frage, wobei allein das geltende Recht untersucht wird. Ausdrücklich wird darauf hingewiesen, daß die Untersuchung nicht versucht, Reformvorschläge zu entwerfen.

Die Arbeit beginnt mit der Darstellung der Grundlagen von kollektiver Sicherheit und Auslegung der Charta durch die Mitglieder und die Organe der Vereinten Nationen. Im Anschluß werden die Befugnisse des Internationalen Gerichtshofs (IGH) anhand der beiden Verfahrensarten, streitiges und gutachterliches Verfahren, sowie die Rechtsprechung des 
IGH, soweit sie Rechtsakte der Organe der Vereinten Nationen betrifft, dargestellt. Des weiteren werden die Handlungsbefugnisse des Sicherheitsrats beschrieben.

Anschließend wird auf die Bindung des Sicherheitsrats an das Recht eingegangen. Insbesondere dieses Kapitel ist für den weiteren Gang der Untersuchung von maßgeblicher Bedeutung. Denn eine gerichtliche Kontrolle des Sicherheitsrats durch den IGH würde von vornherein ausscheiden, bestünde keine derartige Bindung. Dabei wird zunächst die Bindung des Sicherheitsrats an die Charta diskutiert und zutreffend festgestellt, daß ein Organ einer internationalen Organisation zwingend an den Gründungsvertrag gebunden ist. Zudem entbinde der politische Charakter des Sicherheitsrats diesen nicht von einer Bindung an die Charta. Diese allgemein zutreffenden Feststellungen hätten indes hinsichtlich des Sicherheitsrats einer eingehenderen Begründung bedurft. Denn es ist angesichts der Befugnis des Sicherheitsrats, die Charta auszulegen, und der rechtsfortbildenden Praxis seit 1990 nicht ohne weiteres erkennbar, in welchem Umfang der Rat an jede einzelne Vorschrift der Charta gebunden ist. Insofern hätte man sich bereits hier eine eingehendere Untersuchung der Bindung an die Vorschriften der Charta gewünscht. Dies gilt auch für die weitere Untersuchung der Bindung des Sicherheitsrats an humanitäres Völkerrecht und der Rechtsbindung von Zwangsmaßnahmen nach Kapitel VII. Zwar weist der Autor zutreffend darauf hin, daß der Sicherheitsrat sich widersprüchlich verhalten würde, wenn er eine derartige Bindung nicht anerkennen würde. Insofern kommt eine Bindung nach estoppel in Betracht. Jedoch hätte es hier näher gelegen, mit einem Hinweis auf Art. 24 Abs. 2 SVN zu argumentieren, da diese Vorschrift ausdrücklich eine Bindung des Sicherheitsrats an Recht normiert. Ebenso hätte es einer eingehenderen Begründung der behaupteten Bindung des Sicherheitsrats an Normen des ius cogens bedurft. Insofern erscheint die Argumentation des Autors, daß es angesichts der fundamentalen Bedeutung dieser Vorschriften keine Rechtfertigung für eine Abweichung gibt, etwas schwach, läuft sie doch darauf hinaus, daß nicht sein kann, was nicht sein darf. Vielmehr bietet die Charta in Art. 103 SVN eine Grundlage für die Annahme einer Bindung des Sicherheitsrats an ius cogens. Denn diese Vorschrift knüpft allein an vertragliche Pflichten der Mitglieder an und nimmt ungeschriebenes Recht von dem Vorrang der Pflichten aus der Charta aus. Diese angesichts des Umfangs gewohnheitsrechtlicher Vorschriften erstaunliche Regelung bekommt Sinn vor dem Hintergrund, daß Normen des ius cogens insbesondere im ungeschriebenen Recht verankert sind.

Im Anschluß an dieses Kapitel werden die Folgen des rechtswidrigen Handelns untersucht, wobei sich der Autor im wesentlichen der Position des IGH anschließt. Sodann befaßt sich die Untersuchung mit der Frage, welche Institution die Rechtmäßigkeit einer Resolution des Sicherheitsrats feststellen kann. Dabei wird zunächst untersucht, ob diese Kompetenz beim Sicherheitsrat selbst angesiedelt sein kann, was mit dem Hinweis abgelehnt wird, daß eine derartige Kompetenz den Sicherheitsrat zum Richter in eigener Sache machen würde. Ebenso wird eine Prüfungskompetenz der Mitgliedstaaten abgelehnt, da dies das Friedenssicherungssystem der Vereinten Nationen, wie es insbesondere in Kapitel VII ausgestaltet ist, unterlaufen würde, da es ansonsten den Mitgliedern unter Hinweis auf die angebliche 
Rechtswidrigkeit einer Resolution des Sicherheitsrats möglich wäre, deren Befolgung zu verweigern. Nur im Fall of fensichtlicher und schwerwiegender Rechtswidrigkeit solle den Mitgliedstaaten ein right of last resort zustehen. Insofern ist indes anzumerken, daß diese Position die aufgeworfene Frage, wie sich Staaten gegenüber derartigen Resolutionen verhalten sollen, nur annähernd beantwortet. Es wäre insofern angebracht gewesen, deutlicher herauszuarbeiten, anhand welcher Kriterien derartig schwerwiegende und offensichtliche Rechtsverletzungen angenommen werden können, um den Staaten ein right of last resort zugestehen zu können.

Insoweit besteht nach Ansicht des Autors die Notwendigkeit einer gerichtlichen Kontrolle des Sicherheitsrats. Auf diese zentrale Frage geht die Arbeit ein, nachdem zuvor festgestellt wurde, daß die Grundsätze von Litispendenz und res judicata im Verhältnis zwischen Sicherheitsrat und IGH nicht greifen und daß der IGH durchaus die Kompetenz hat, über politische Fragestellungen zu entscheiden. Zunächst werden die verfahrensrechtlichen Möglichkeiten einer gerichtlichen Prüfung untersucht. Es wird festgestellt, daß sowohl das streitige Verfahren als auch das gutachterliche Verfahren Grundlage für eine derartige Prüfung sein können. Sowohl eine prinzipale Prüfung, d.h. eine Resolution des Sicherheitsrats wird Gegenstand des Verfahrens, als auch eine inzidente Prüfung, d.h. die Rechtmäßigkeit einer Resolution wird Vorfrage des Verfahrens, sind zulässig. Leider läßt die Arbeit insofern eine eingehende Begründung vermissen. Hinsichtlich einer prinzipalen Prüfung im streitigen Verfahren wird deren Zulässigkeit mit dem einfachen Hinweis auf das Lockerbie-Verfahren bejaht. In diesem Verfahren hätte Libyen die Feststellung, daß die Resolution 748 (1992) rechtswidrig sei, beantragen können. Diese Argumentation macht indes den Sicherheitsrat praktisch zur Partei des Verfahrens, was mit der ausschließlichen Parteifähigkeit von Staaten unvereinbar wäre. Weniger zweifelhaft ist dagegen die Annahme der Zulässigkeit einer inzidenten Prüfung im streitigen Verfahren. Diese Verfahrenskonstellation begegnet nicht dem Einwand der ausschließlichen Parteifähigkeit von Staaten und findet zudem eine Grundlage in der umfassenden Prüfungs- und Rechtsanwendungskompetenz des IGH. Ebenso ist die Annahme einer Prüfungskompetenz im gutachterlichen Verfahren nicht ausreichend. Die Arbeit argumentiert insofern allein mit einem Hinweis auf die Rechtsprechung des IGH. Indes bietet Art. 96 SVN eine ausreichende Grundlage, um eine Prüfkompetenz des Sicherheitsrats annehmen zu können. Nach dieser Vorschrift kann der IGH jede Rechtsfrage beantworten, die eines der vorlageberechtigten Organe dem Gerichtshof vorlegt. Bereits der Wortlaut der Vorschrift stützt diese Auffassung. Hinzu kommt, daß es dem Zweck der Vorschrift, den Vereinten Nationen legal guidance an die Hand zu geben, widersprechen würde, wenn die Prüfungskompetenz des IGH derart eingeschränkt wäre, daß Resolutionen des Sicherheitsrats per se ungeprüft angewendet würden.

Zutreffend wird im folgenden erörtert, daß weder das streitige Verfahren noch das gutachterliche Verfahren geeignet sind, den Sicherheitsrat an die Entscheidung des IGH zu binden. Gem. Art. 59 IGH-Statut bindet die Entscheidung nur inter partes; gemäß Art. 34 IGH-Statut können nur Staaten Partei im streitigen Verfahren werden. Hieraus folgt zwin- 
gend, daß der Sicherheitsrat als Organ einer internationalen Organisation nicht durch eine streitige Entscheidung des IGH gebunden werden kann. Dies gilt auch für ein Gutachten, da dieses als "Ratschlag" nicht verbindlich ist. Insofern kommt nach Ansicht des Autors nur eine faktische Bindung des Sicherheitsrats in Betracht. Denn der IGH stelle die geltende Rechtslage fest, von welcher der Sicherheitsrat nicht abweichen werde. Diese Auffassung ist in dieser Form nicht überzeugend, da es wahrscheinlich ist, daß der Sicherheitsrat seine eigene, vom IGH abweichende Rechtsauffassung vertreten und begründen wird. Insofern hätte es bei dieser entscheidenden Frage einer weitergehenden Auseinandersetzung mit den politischen Auswirkungen einer Entscheidung des IGH bedurft. Hier wäre es durchaus denkbar gewesen zu argumentieren, daß dieser Konflikt zu Lasten des Sicherheitsrats gehe, da der IGH als neutraler, weniger den Interessen der Mitglieder verpflichtetes Organ gröBere Autorität genießt.

Des weiteren wird erörtert, ob eine gerichtliche Prüfung vor dem Hintergrund einer potentiellen Kontrolle durch den Sicherheitsrat und die Generalversammlung ausgeschlossen ist. Dies wird unter Hinweis auf Art. 11 und 12 SVN für die Generalversammlung verneint. Für den Sicherheitsrat wird im Hinblick auf Art. 27 Abs. 3 SVN eine interne Kontrolle angenommen. Jedoch könne hieraus nicht gefolgert werden, daß diese Form der Kontrolle einen Anspruch auf Exklusivität erheben könne. Ebenso könne gegen eine gerichtliche Prüfung nicht die Friedenssicherungskompetenz des Sicherheitsrats aus Art. 24 SVN eingewendet werden, da der Entscheidung des IGH insofern keine aufschiebende Wirkung zukomme. Zwar trifft es zu, daß Entscheidungen des IGH keine aufschiebende Wirkung entfalten. Jedoch ist diese Argumentation insofern wenig überzeugend, da - wie der Autor zuvor selbst festgestellt hat - Entscheidungen des IGH faktische Auswirkungen haben. Insofern kann nicht allein mit dem Fehlen aufschiebender Wirkung der Entscheidung argumentiert werden. Zudem ist zu berücksichtigen, daß bereits die Anhängigkeit eines Verfahrens, welches die Rechtmäßigkeit einer Entscheidung des Sicherheitsrats betrifft, die Befolgung einer derartigen Resolution in Zweifel ziehen kann, da den Staaten das Argument an die Hand gegeben wird, daß sie der Resolution erst nach der Entscheidung des Gerichtshofs Folge leisten werden. Angesichts der Verfahrensdauer vor dem IGH ist dies eine ernstzunehmende Einschränkung der Friedenssicherung durch den Sicherheitsrat.

Im letzten Abschnitt befaßt sich die Untersuchung mit der Kontrolldichte, die insbesondere anhand der Spielräume des Sicherheitsrats nach Kapitel VII abgehandelt wird.

Insgesamt überzeugt die Arbeit insofern, als die maßgeblichen Fragen angesprochen und zu überzeugenden Ergebnissen geführt werden. Insbesondere die Darstellung von bestimmten prozessualen Situationen, in welchen die Rechtmäßigkeit von Resolutionen des Sicherheitsrats relevant werden kann, stellt einen wesentlichen Beitrag zur Diskussion dar. Die Arbeit leidet jedoch daran, daß sie eine tiefergehende Argumentation verschiedentlich vermissen läßt.

Nils Meyer-Ohlendorf 